\title{
Research on the Database Design of Business Management System
}

\author{
Jian YU \\ Education Technology College \\ Shenyang Normal University \\ Shenyang,China \\ davidyu0566@sina.com
}

\author{
Yu YAN \\ Software College \\ Shenyang Normal University \\ Shenyang, China \\ ybrady1234@126.com
}

\begin{abstract}
Business management is an important part of enterprise management. Information management system is an important means to realize the modernization of management, goods. The current problems, the construction of business management information system, the design of database, database management system in this paper support, according to the database design process of the conceptual structure design, logical structure design and physical structure design. The performance of the E-R model through the conceptual structure design. Part of the design choice of structure design logical structure of data types and relationships. Selection and index design physical structure design part of the disk hardware. The content of this paper, provides the enterprise for the development of business management system to strengthen the practical solution of business management, has an important significance to improve the level of information.
\end{abstract}

Keywords- database design; business; management system; structure design ; relation design

\section{INTRODUCTION}

Business management is an important part of enterprise management, the central link in enterprise management. Speaking of the enterprise, rational procurement, timely sales, reduce the backlog, ensure a reasonable inventory level, and an effective way to obtain better economic benefits.

In the case of enterprise management of the artisans, the enterprise is very difficult to obtain timely inventory, sales statistics, the production department is difficult to make a reasonable production plan, prospect and market demand of production is difficult to accurately reflect the production enterprises.

With the development of global economic integration and the development of electronic commerce, the competition between enterprises will be more and more fierce, the rapid development of network and e-commerce breakthrough in time and space constraints, bring more development opportunities. The basic work of construction of electronic commerce database enterprise, construction only reasonable and effective database system, the development of enterprise information management system, in order to realize the electronic commerce.

Database design the basic database, the database design is the application of a given, the optimal structure model of database, establishment and application of database, so as to effectively store data, to meet the application requirements of various users [1].

\section{CONCEPTION DESIGN RESEARCH}

The concept of structural design is also known as the conceptual design, the user needs a abstract concept model. The logical structure of the conceptual data model database independent, independent of the database management system of concrete(DBMS) is the real world of intermediary and the machine world. The most commonly used tool, conceptual structure design is entity relationship diagram (E-R), E-Rdiagram represent entities, attributes, relations, the conceptual model is used to describe the real world.

Constitute the basic elements of E-R graph of entities, attributes and relationships, solid rectangle, rectangle filling property name in heentity; elliptic, elliptic filling property name, using undirected edges are connected to the corresponding entity relation; diamond, no relationship between name and undirected edge connecting the real diamond filled, standard type the next (1: 1, $1: \mathrm{n}$ or $\mathrm{m}: \mathrm{n}$ ). The conceptual design of E-R model of the system is shown in Fig. 1 [2].

\section{LOGICAL DESIGN RESEARCH}

\section{A. Type Selection Research}

In Database, there are 24 multiple data types, based on a conceptual model of the business management system, grouped into five types of data, namely:

\section{1) Character type}

The character data of letters, symbols and numbers any combination, including the type of Char, Varchar, and Text, etc. Varchar is a variable-length character data, the length does not exceed $8 \mathrm{~KB}$; Char is a fixed-length character data, the length does not exceed $8 \mathrm{~KB}$; More than $8 \mathrm{~KB}$ ASCII data can use the Text data type is stored. The character data type selection varchar.

2) Integer type

The integer stored data type is Int, Smallint, and Tinyint. The Int type storage data range is greater than the range of data types are stored in Smallint, and the Smallint 
type storage data range greater than the range of Tinyint types of stored data.

The Int type a value to occupy 4 bytes of storage space, Smallint type each value occupies 2 bytes of storage space, Tinyint type a value occupies 1 byte of storage space. The system select Smallint integer type.

3) Precise decimal type

The precise decimal data type in Database Decimal and Numeric data storage space occupied by Integer digits and the number of decimal places to determine, on either side of the decimal point store 38. Requires higher precision of the right of the decimal point, the remaining used to represent the integer part of the number, the less. The exact decimal type select Decimal.

\section{4) Currency type}

Currency data type Money and Smallmoney, Money type occupies 8 bytes of storage, Smallmoney data type occupy 4 bytes of storage. The currency type Smallmoney.

5) Date Time type

By the effective date and time, the date time data types include Datetime and Smalldatetime two types. Datetime type occupies 8 bytes of storage, date range from ending 9999-12-31 to 1753-1-1 occupy 4 bytes of storage; Smalldatetime type, date range is from 1900-1-1 to 2079 12-31 end. The system date time type select Smalldatetime [3].

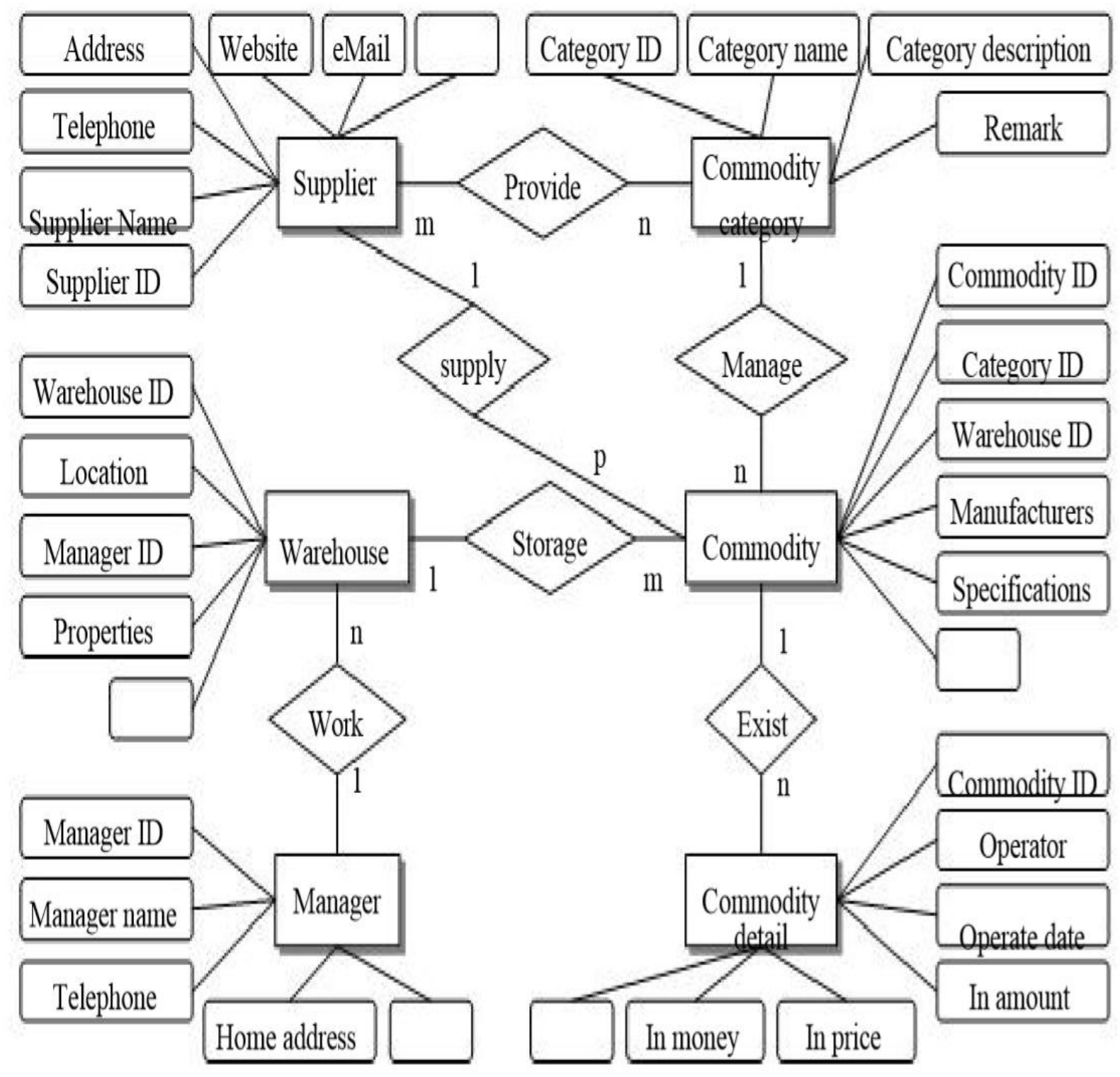

Figure 1. E-R diagram of commodity management system 


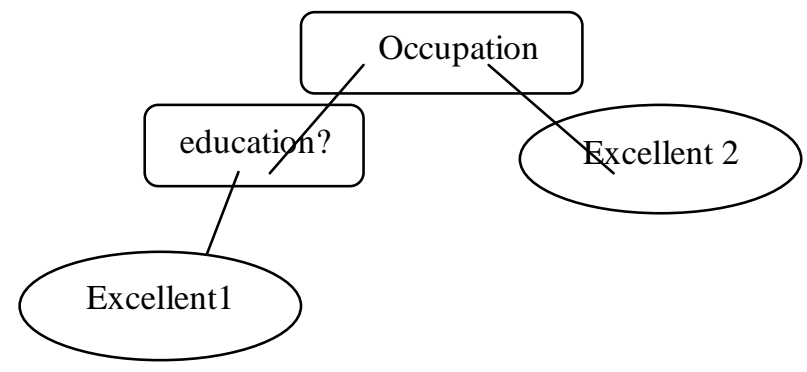

Figure 2. Relation management system

\section{B. Relational Design Research}

Fig .1 and Fig .2 exist two relationship types, one-tomany (1:n) and many-to-many (m:n), according to the logical structure design principles : an entity is converted to a relational schema, the entity attribute is the attribute of relationship, the entity code is the code of relationship.

A 1:n relationship can be converted into a separate relational schema, with the $\mathrm{N}$-terminal corresponding relational schema merger, if converted to an independent relationship model code of each entity connected with the relationship and relationship attribute converted to the attribute of the relationship, and the relationship code is the code of the $n$ - terminal entities; an $m: n$ relationship converted into a relational schema, code of various entities connected to the relationship and relationship attribute is converted to the attribute of the relationship, and the relationship between the code combination of the code for each entity. The logical structure design results are as follows:

Supplier table (Supplier ID, Supplier Name, Contact person, Charge person, Telephone, Address, Post code, Website, eMail, Deposit bank, Bank account number, Credit grades, Remark) Commodity category table (Category ID, Category name, Category description, Remark) Provide table (Supplier ID, Category ID, Remark) Commodity table (Commodity ID, Commodity name, Category ID, Warehouse ID, Manufacturers, Purchase price, Sales price, Specifications, Measurement unit, Remark) Commodity detail table (Commodity ID, Operator, Operate date time, In amount, In price, In money, Out amount, Out price, Out money, Balance amount, Balance price, Balance money, Remark) Warehouse tables (Warehouse ID, Warehouse name, Location, Manager ID, Volume, Properties, Ware location amount, Remark) Manager tables (Manager ID, Manager name, Sex, Nation, Age, Home address, ID number, Professional title, Duty, Telephone, Remark) $[9,10]$.

\section{PhysiCAl DESIGN RESEARCH}

\section{A. Disk Hardware Selection}

The performance parameters of the hard disk, hard choice for access to the database from the capacity, speed, cache and other aspects to consider. Business management system more amount of data, you need to choose a dedicated database server, to consider a RAID device. RAID disk system is composed of multiple disk drives can provide higher performance, reliability, storage capacity and lower cost. Fault-tolerant array is divided into 0-5 of 6 RAID level. Each level uses different algorithms to achieve fault-tolerant [4] .

\section{B. Index Design}

When no index on the column is accessed, the row of data obtained from a table of specific conditions will be a full table scan operation, the server processes all data blocks in the table is loaded into memory, and then progressive match, then the main SQL execution time consumption in the table data loading and data matching, you need to consume a large amount of the cost of IO and CPU cost [5].

The index is to sort the structure of the database table one or more column values, creating an index can improve system performance. By creating a unique index to ensure the uniqueness of the data of each row in the database table; Accelerate the speed of data retrieval, which is also the main reason to create the index; Connection between the table and table acceleration, particularly in achieving the integrity of the data reference particularly meaningful; Grouping and sorting clause for data retrieval, the same significant reduction in the time of the query grouping and sorting; Hidden by the index query optimization, to improve system performance [6].

In this system, "Supplier table" creating a clustered index by "Supplier ID", non-clustered index created by the "Supplier Name, Contact person, Charge person"; "Commodity category table" to create a clustered index by "Category ID", "Category Name" to create a non-clustered index; "Provide table" to create the clustered index by "Supplier ID, Category ID"; "Commodity table" clustered index created by the "Commodity ID", "Commodity name, Category ID, Warehouse table, Warehouse ID, Manufacturers" to create a non-clustered index; "Commodity detail table" to create the clustered index by "Commodity ID, Operate date time", "Operator" to create a non-clustered index; "Warehouse table" create a clustered index by "Warehouse ID", non-clustered index created by the "Warehouse name, Location, Manager ID"; "Manager table" create the clustered index by " Manager ID", "Manager name, Sex, Age, ID number, Professional title, Duty" to create a non-clustered index [7]. 


\section{CONCLUSION}

With the development of information technology, computer data processing has occurred Profound changes, the database is a widely used client / server database management system. Architecture, using transact SQL language, transfer of SQL between the client and the server, the client.

A request to the server to handle the business environment of the results meet the requirements. Different types of database solution. There are many obvious advantages: ease of use, scalability.

The distributed organization, a data warehouse for decision support, and other closely related. Integration server software, with high performance and low cost. In this paper, based on the database design, and provides practical solutions business management system, enterprise. Strengthen enterprise management, has the vital significance to improve the level of information [8].

\section{REFERENCES}

[1] Jian. YU, " Study of Insurance Information base on Data Mining " , Vehicle,Mechatronics and Information Technologies, 2013 .
[2] Y. L. Yu, "CS-based Merchandise Management Systems Architecture and Implementation of Research," Master's degree of Ocean University of China, 2011.

[3] H. M. Chen, "Logical structure design of database," Fujian Computer, vol. 28, no. 10, pp. 214-215, 2012.

[4] S. Wang, S. X. Sa, "Introduction to database system (Fourth Edition)," Higher Education Press, 2013

[5] L. J. Zheng, K. Li "Database Design of Materials Classification Detailed Account," Lecture Notes in Information Technology, vol. 1, pp. 104-108, 2012.

[6] J. S. Yang, C. Zhang, "Impact of index on the SQL execution costs," Information \&Communications, vol. 27, no. 1, pp. 136-137, 2013.

[7] K. Li, "Column stores the index technology of SQL Server 2012," Computer CD Software n and Applications, vol. 15, no. 19, pp 121-123, 2012.

[8] C. Chen "Establishment and analysis on index of SQL Server," Science \& TechnologyInformation, vol. 29, no. 36, pp. 288-289, 2012

[9] [Chih-Fong Tsai, Yu-Hsin Lu, David C. Yen, "Determinants of intangible assets value: The datamining approach", KnowledgeBased Systems, Vol. 31, pp. 67-77, 2012.

[10] David L. Olson, Dursun Delen, Yanyan Meng, "Comparative analysis of data mining methodsfor bankruptcy prediction", Decision Support Systems, Vol. 52, No. 2, pp. 464-473, 2012 